Annuaire suisse de politique de développement

\title{
Logique de l'accumulation versus logique de développement : les enjeux des nouveaux partenariats public-privé
}

Frédéric Lapeyre

\section{OpenEdition}

Journals

Édition électronique

URL : http://journals.openedition.org/aspd/338

DOI : 10.4000/aspd.338

ISSN : 1663-9669

Éditeur

Institut de hautes études internationales et du développement

Édition imprimée

Date de publication : 1 octobre 2005

Pagination : 23-41

ISSN : $1660-5934$

\section{Référence électronique}

Frédéric Lapeyre, «Logique de l'accumulation versus logique

de développement : les enjeux des nouveaux partenariats public-privé », Annuaire suisse de politique de développement [En ligne], 24-2 | 2005, mis en ligne le 18 février 2010, consulté le 08 septembre 2020. URL : http://journals.openedition.org/aspd/338; DOI : https://doi.org/10.4000/aspd.338 


\title{
Logique de l'accumulation versus logique de développement: les enjeux des nouveaux partenariats public-privé
}

\author{
Frédéric Lapeyre*
}

a «doctrine Truman », telle qu'énoncée dans le point IV du discours présidentiel sur l'état de l'Union de 1949, a marqué profondément et durablement la coopération au développement. Truman traduisait alors dans son discours la vision dominante du développement comme processus de modernisation devant assurer la transformation de sociétés traditionnelles en sociétés modernes. Cette vision dichotomique «traditionnel versus moderne» va avoir des conséquences très importantes sur la place des acteurs non étatiques et, en particulier, des acteurs populaires dans la définition des politiques de développement. L'acteur central du développement est clairement défini, c'est l'Etat qui, aux mains d'élites modernisatrices - qu'il convient d'appuyer contre les élites radicales révolutionnaires -, doit lancer des politiques volontaristes de développement économique; il y a à cette époque un très large consensus sur l'importance de la planification du développement comme un outil essentiel de transformation socio-économique.

Le rôle de la coopération au développement était, dans ce cadre, de soutenir les pays ou régions «sous-développés » à travers l'aide financière et technique afin de permettre leur décollage économique et d'accélérer leur processus de rattrapage. Les populations et, en particulier, les acteurs populaires étaient perçus dans ce cadre comme des non-acteurs du développement, des porteurs de valeurs traditionnelles qui étaient autant d'obstacles à la modernisation, des «pauvres» passifs et ignorants en attente d'assistance extérieure qu'il fallait absolument aider à sortir de leur misère. Ils devenaient une population cible pour la coopération au développement et, à ce titre, ils ont été soumis à d'incessantes vagues de politiques visant à les moderniser et à les développer, afin d'assurer leur convergence vers des critères universaux de modernité définis à partir de l'expérience historique occidentale de développement.

Au début des années 1980, la contre-révolution néolibérale a bouleversé la vision dominante du rôle de l'Etat dans les politiques de développement. Le secteur public perd son statut de moteur du développement au profit du secteur privé, qu'il soit domestique ou transnational, auquel il revient d'assurer le retour de la croissance. Les exigences de l'international priment dorénavant sur le national et celles du secteur privé sur celles du secteur public. La prise de conscience, dans les années 1990, des excès de ce que l'on a appelé le «consensus de Washington» va se traduire par une réhabilitation de l'Etat, dont on reconnaît le rôle positif à condition qu'il mette en œuvre les principes de bonne

* Professeur à l'Institut d'études du développement, Université catholique de Louvain. 
gouvernance. Les quatre grands principes de la coopération au développement deviennent alors la participation, les partenariats public-privé, l'appropriation et l'empowerment.

La participation apparaît aujourd'hui comme la recette magique des politiques de développement, tout comme l'ajustement structurel et la libéralisation dans les années 1980 et 1990. Elle constitue un des éléments centraux du «cadre intégré de développement» et des stratégies de réduction de la pauvreté, prônés par la communauté internationale (Banque mondiale 2001). La réorientation de la coopération au développement au profit des acteurs non étatiques et les nouvelles formes de conditionnalité liées à la bonne gouvernance ont soutenu les processus de démocratisation et de décentralisation dans les années 1990 au Sud. Cette évolution a créé un contexte propice au développement de nouveaux partenariats, qui s'est traduit par un élargissement du champ de compétences du secteur privé, des organisations de la société civile et des autorités locales.

Pour comprendre les enjeux des transformations récentes de la coopération au développement, il est nécessaire de revenir sur les grandes étapes de son évolution et d'analyser les nouveaux dispositifs qui ont été mis en place ces dernières années. Ceux-ci - notamment l'Accord de Cotonou et les documents de stratégie pour la réduction de la pauvreté (DSRP) - matérialisent le nouveau paradigme du développement qui a profondément bouleversé la distribution des rôles et des responsabilités des acteurs étatiques et non étatiques. Pour finir, nous mettrons l'accent sur les risques et les opportunités associés à ce nouveau cadre de la coopération au développement pour les organisations populaires.

\section{La place de l'Etat au début de la coopération au développement}

Si l'on jette un regard critique sur l'économie du développement telle qu'elle s'est constituée dans les années 1940-1950, on constate qu'elle est empreinte d'un pessimisme important quant aux résultats du laisser-faire des mécanismes de marché, une vision volontariste du développement et un grand optimisme concernant la planification du développement. La mise en lumière des défaillances du marché légitimait l'intervention publique directement dans la sphère de la production - la coordination et l'administration du secteur public n'étant pas considérées comme problématiques (Tinbergen 1984:326). La théorie normative de l'Etat, qui a été dominante jusqu'au début des années 1970, prévoyait donc que là où il y avait des défaillances du marché, l'Etat devait y remédier au moyen d'interventions appropriées et de ce fait aller améliorer le bien-être de la communauté dans son ensemble.

La coopération au développement a été très marquée par cet optimisme systémique quant à l'intervention publique, qui comportait trois lacunes importantes: premièrement, l'insuffisance de réflexion sur le problème de l'interaction Etatmarché dans le cadre d'une économie mixte (la nécessité de combiner de manière efficace les forces et faiblesses de chacun étant ignorée), deuxièmement, une conception simpliste de la nature de l'Etat et de l'impact de ses politiques et, troisièmement, la non intégration de la société civile dans le processus décisionnel. Or, c'est dans ces brèches que vont s'engouffrer plus tard les économistes néolibéraux et autres pour miner le rôle de l'Etat dans le développement. 
La victoire politique et académique de la pensée néolibérale dans les années 1980 s'est traduite par un changement profond de la perception de l'Etat chez beaucoup de ceux qui réfléchissaient sur les politiques de développement. A la vision quelque peu naïve de l'Etat bénévole entretenue par les pionniers de la coopération au développement, la nouvelle économie politique (NEP) a substitué la vision extrêmement cynique d'un Etat prédateur et corrompu. Dans ce cadre, chaque groupe d'intérêts spécifiques - politiciens, électeurs, fonctionnaires, syndicats, producteurs domestiques, etc. - cherche à manipuler 1'Etat à son avantage afin de maximiser son propre bien-être au détriment de celui de la société dans son ensemble. Toute l'attention s'est portée sur le problème de l'accroissement du poids de l'Etat sur l'économie, qui, en stimulant le processus concurrentiel de recherche de rentes des agents aussi bien privés que publics, était vu comme la source de toutes les distorsions dont souffraient aussi bien les Etats keynésiens du Nord que les Etats volontaristes du Sud et les systèmes socialistes à l'Est (Krueger 1990:426).

Les effets pervers de l'intervention publique étaient dénoncés: secteur public irrationnel et coûteux, distorsions dans les structures de production et de distribution, politique dirigiste des prix inefficiente, etc. Les néolibéraux mettaient en avant que les coûts de l'interventionnisme dépassaient les bénéfices et que, quitte à choisir, les défaillances du marché étaient moins dommageables que celles de l'Etat. A ce titre, toute tentative de l'Etat de remédier au problème de la pauvreté était toujours perçue par la NEP comme devant conduire à une aggravation de ce problème du fait des effets pervers induits par son intervention. Selon la NEP, le volontarisme de l'Etat dans l'économie conduisait à un développement des activités économiques légales et illégales destinées à capter des rentes générées par l'activité administrative publique. L'Etat ne devait donc plus être considéré comme hypercompétent et cherchant à maximiser le bienêtre général mais comme le résultat d'une interaction complexe de groupes d'intérêts. Ainsi, il était proposé une théorie unifiée des motivations humaines selon laquelle les choix rationnels des agents économiques individuels - qu'ils appartiennent au secteur public ou bien privé - obéissent à l'hypothèse de maximisation de leur intérêt personnel (Tollison 1982).

La solution avancée par ce nouveau courant de pensée dominant était de ramener l'Etat à sa taille minimale et de revenir au libre jeu des mécanismes de marché dans l'allocation des ressources. Il est clair que la NEP a largement imprégné les programmes d'ajustement structurel et de réforme, proposés par les institutions internationales dans les années 1980 et 1990. Le rapport Berg de la Banque mondiale, publié en 1981, en est une parfaite illustration puisqu'il attribue la crise du développement en Afrique à l'intervention publique excessive qui aurait abouti à un sous-développement de l'agriculture et du secteur d'exportation (Banque mondiale 1981). Le thème central de ce rapport - dont la philosophie s'apparente à celle de la NEP - porte sur l'inefficacité et le coût de l'Etat en Afrique, ce qui suppose, au niveau de l'économie politique de la réforme que l'on réduise l'Etat et que l'on développe et soutienne les capacités managériales du secteur privé. 
L'un des objectifs principaux des organisations internationales à travers les programmes d'ajustement structurel était d'imposer une redéfinition du partage des rôles entre secteur privé et secteur public (Jolly, Emmerij, Ghai et Lapeyre 2004). L'Etat devait rationaliser le secteur public en réévaluant ses activités en fonction de critères de bonne gestion et d'allocation efficiente des ressources rares. La doctrine de l'ajustement structurel préconise notamment un plus grand rôle attribué au système de prix dans l'allocation des services essentiels (éducation, santé, transport, eau, énergie, etc.), car ceux-ci se sont révélés coûteux et sources de gaspillage lorsqu'ils étaient aux mains de l'Etat; elle encourage de ce fait leur privatisation (Colclough 1992:199). D'autre part, la réduction des activités parastatales - spécialement dans les activités directement productives et les sous-secteurs potentiellement profitables que sont le commerce et les transports - devait créer des opportunités pour les entrepreneurs dynamiques et flexibles du secteur privé.

Dans le nouveau modèle de croissance proposé, l'Etat ne devait plus mener de politiques volontaristes reposant sur une stratégie nationale de développement car celles-ci s'étaient soldées par des échecs. L'accent était mis sur la réduction du secteur public et des investissements publics au profit de la stimulation des forces de marché à travers la mise en place d'un vaste dispositif visant à offrir aux opérateurs privés l'environnement socio-économique le plus favorable à la poursuite de leurs activités. L'Etat devait libéraliser l'économie et se retirer des activités dans lesquelles il était traditionnellement engagé, excepté dans le cas des infrastructures économiques destinées au secteur privé. La responsabilité de l'Etat quant à l'accès du plus grand nombre aux services essentiels de base était remise en question au nom de la rationalité économique, qui exigeait la privatisation d'une part importante de ces services et l'augmentation de leurs tarifs, qu'ils aient été privatisés ou non.

On peut noter, à ce titre, le caractère normatif très marqué du discours sur la décentralisation et la promotion des acteurs décentralisés (y compris le discours dominant sur la promotion du secteur informel). Ces objectifs sont cohérents avec l'offensive néolibérale, amorcée à la fin des années 1970, de délégitimation de l'Etat et de désarticulation des solidarités nationales. Les processus de déconcentration et la décentralisation ont affaibli la capacité des Etats à mener des politiques de développement nationales. On a assisté à une profonde remise en cause de l'Etat comme garant de la cohésion sociale et de l'aménagement du territoire au sens large et à une redéfinition de son rôle à partir des contraintes de la logique des pôles performants et de la nécessaire gestion des processus de fragmentation sociale et spatiale qui y sont associés (Peemans 1997).

Le démantèlement du secteur public, la baisse de l'emploi formel (résultant des privatisations et de l'ouverture de l'économie à la concurrence internationale) ainsi que les politiques budgétaires restrictives ont affaibli les porteurs du projet national de modernisation au profit des couches sociales qui ont vu dans l'application des programmes d'ajustement structurel le moyen d'accroître leur pouvoir. En les considérant comme les agents positifs de la croissance, les programmes d'ajustement structurel ont contribué à renforcer les élites au Sud liées au secteur privé et favorables à l'intégration dans l'économie mondiale. Le pré- 
sident de la Banque mondiale, James Wolfensohn, reconnaissait lui-même ce nouveau contexte en déclarant: «Un autre changement majeur est intervenu: les employeurs, les investisseurs ou le secteur privé, comme nous les appelons, occupent aujourd'hui une place radicalement différente de celle qu'ils occupaient il y a sept ans. Le secteur privé était alors deux fois moins important que le secteur public. Il représente aujourd'hui 240 milliards de dollars, soit cinq fois plus que le secteur public. Ce n'est pas seulement un changement quantitatif; c'est un saut qualitatif. Nous avons un nouvel acteur, un acteur de poids, dans le domaine du développement...» (Wolfensohn $1997: 2$ ).

\section{Le nouveau paradigme du développement dans les années 1990}

A la fin des années 1980, un large consensus va émerger sur les coûts sociaux dramatiques des programmes d'ajustement structurel et leurs résultats décevants en termes de retour à la croissance. La publication par l'UNICEF en $1987 \mathrm{du}$ livre intitulé L'ajustement à visage humain, puis la propagation de préoccupations de développement humain dans les autres institutions internationales de développement, ont marqué l'avènement du nouveau consensus sur la lutte contre la pauvreté autour duquel allait s'articuler le discours dominant dans les années 1990 (Cornia, Jolly et Stewart 1987; UNDP 1990; World Bank 1990).

Dans le cadre de cette évolution, la présentation faite par Joseph Stiglitz en 1998, lors du cycle de conférences Raúl Prebisch organisé par la CNUCED, mérite une attention toute particulière. Il traduit parfaitement le «consensus post-Washington» sur le processus de transformations socio-économiques à entreprendre; celui-ci reposait sur quatre éléments principaux: l'articulation entre marché et Etat avec le secteur privé comme moteur de la croissance, l'intégration dans l'économie mondialisée, la démocratisation et la bonne gouvernance.

Au nom de la Banque mondiale, Stiglitz a effectué en 1999 un véritable mea culpa pour le type de conditionnalité imposé dans les années 1980. S'appuyant sur leurs rapports d'évaluation internes des projets et programmes de développement qu'elles avaient financés, les organisations internationales ont reconnu que toute tentative d'imposer le changement de l'extérieur court des risques élevés d'engendrer des résistances à l'intérieur et de créer des barrières au changement au lieu de le faciliter (World Bank 1998; Isham, Narayan et Pritchett 1995). Dès lors, la mise en place de mécanismes participatifs lors du processus de formulation de la stratégie de développement doit permettre de construire un consensus autour de la stratégie élaborée et, de cette manière, stimuler un processus d'appropriation des réformes par les populations afin d'accroître leurs chances de succès et réduire les risques d'instabilité sociale et politique (Stiglitz 1998: 14).

Les déclarations récentes de Wolfensohn sont en cela très représentatives: «Le développement n'est pas une question de charité. C'est une question d'inclusion et de renforcement des capacités... Les populations victimes de la pauvreté veulent prendre la parole», ou encore «La participation est un gage de résultats tangibles au niveau des projets et des programmes. Et elle peut favoriser le consensus, qui est le fondement même de l'évolution et de la refonte du cadre social» (Wolfensohn 2000). Le Rapport sur le développement dans le monde 2000/2001 de la Banque mondiale, intitulé Combattre la pauvreté, adopte explicitement 
l'idée d'empowerment à la fois comme dimension inhérente du développement et comme moyen de lutter contre la pauvreté.

L'intérêt grandissant pour le concept de capital social dans les années 1990 est aussi associé à la reconnaissance du rôle fondamental de la société civile et des médiations sociales plus ou moins institutionnalisées entre l'Etat, le marché et la société civile dans les processus de développement (Woolcock 1998:155). Le concept de capital social fait référence au stock de normes de réciprocité et de confiance ainsi qu'aux réseaux d'engagement civique qui sont des caractéristiques de l'organisation sociale (Putnam 1993a: 167). Ceux-ci peuvent améliorer l'efficacité de la société en facilitant des actions coordonnées, d'où l'importance de ce stock de capital social dont va dépendre le degré de coopération dans la société. La prise en compte du capital social, au côté du capital physique et du capital humain, résulte du constat de l'importance du stock de capital social d'une communauté afin de lancer des initiatives de développement cohérentes, efficaces et viables. La création d'un environnement favorable au développement est précisément le fruit de cet ensemble de liens sociaux qui lient l'Etat à la société et un tel environnement fournit des canaux institutionnalisés permettant une négociation continuelle des objectifs et des politiques (Evans 1995:164).

Si, pour le courant libéral, l'intervention de l'Etat est inappropriée pour accroître le capital social et, au contraire, conduit à sa destruction (Coleman 1990; Fukuyama 1995), une part importante de la littérature sur le capital social insiste sur l'idée de synergies. L'hypothèse de synergies suppose que l'Etat contribue à un environnement favorable à l'engagement de la société civile dans le processus de développement à travers l'instauration d'un climat de confiance et la stimulation de réseaux réunissant les différents acteurs concernés, dont la mobilisation assurera en retour l'efficacité et la viabilité des politiques de développement (Putnam 1993b : 42). Evans insiste sur le potentiel de développement d'une combinaison d'institutions publiques fortes et de communautés organisées permettant d'exploiter au mieux les complémentarités de chacun (Evans 1996: 130). L'Etat joue un rôle particulièrement important dans la reproduction du capital social du fait que celle-ci est tributaire de toutes les institutions visant à favoriser les échanges et à accroître le niveau de coopération. C'est donc bien d'un jeu à somme positive dont il est question ici.

\section{De la promotion des partenariats public-privé}

Si, traditionnellement, l'Etat avait assumé la responsabilité de l'accès de la population aux services essentiels, on assiste au Sud depuis deux décennies à une croissance importante du rôle du secteur privé dans la santé et l'éducation et à une tendance à la privatisation de l'approvisionnement en eau et en électricité et des services d'assainissement. Les raisons avancées pour cette évolution sont de trois ordres: la faiblesse des ressources publiques, la médiocre qualité du service public et les pressions en faveur de la libéralisation économique. L'idée dominante est qu'un service public ne constitue pas toujours la meilleure solution lorsque les institutions sont peu solides et que l'utilisation des fonds publics manque de transparence (UNDP 2003). Le problème identifié par les organisations de Bretton Woods, entre autres, est donc que beaucoup trop de services publics sont mal gérés par les autorités publiques responsables. De plus, souf- 
frant d'un manque de ressources aigu, nombre d'Etats des pays pauvres ne peuvent garantir des services collectifs efficaces et universels, ni financer d'importants investissements d'infrastructure pour la maintenance et le développement de ces services. Cette situation est un obstacle majeur à la réalisation des Objectifs du millénaire pour le développement formulés par les Nations unies, dont notamment celui de réduire de moitié d'ici à 2015 la proportion de personnes n'ayant pas accès à une eau potable saine.

C'est pourquoi la Banque mondiale insiste systématiquement pour que les Etats s'engagent dans la recherche d'une plus grande efficacité dans l'utilisation des fonds publics et d'un meilleur rapport qualité-prix de ces services essentiels. La poursuite de ces objectifs s'est traduite soit par la privatisation de ces services essentiels, soit par un changement radical du mode de gestion publique de ces services. D'une part, la privatisation a permis de supprimer les subventions aux entreprises publiques déficitaires qui pèsent sur les dépenses publiques (priorité des organisations de Bretton Woods) car, lorsque les infrastructures sont publiques, les tarifs sont souvent trop bas pour permettre le recouvrement des coûts et les problèmes de non-paiement sont fréquents. La privatisation était aussi censée améliorer l'efficacité, la productivité et la profitabilité dans le secteur des services essentiels en y introduisant la rationalité et le dynamisme des forces de marché dans la prise de décision et la gestion des affaires. D'autre part, même lorsque ces services n'étaient pas privatisés, les nouvelles règles de gestion publique reposent sur le principe de la couverture totale des coûts, qui impose aux prestataires de services de «viser un niveau de revenus permettant de couvrir l'intégralité de leurs dépenses récurrentes, tout en élaborant une politique durable de couverture des coûts, qui tienne compte des besoins futurs en trésorerie» (Camdessus 2003 : 24). Dans les deux cas - privatisation et nouvelle gestion publique -, les populations les plus pauvres ont dû affronter une augmentation substantielle des coûts et des problèmes grandissants d'accès.

Dans les années 1980 et 1990, les nouvelles élites dominantes ont relayé les demandes des forces de marché relatives à la libéralisation économique et à l'ouverture des marchés, qui ont conduit à l'essor de prestataires privés pour les services collectifs essentiels. Les bailleurs de fonds ont aussi joué un rôle important dans ce sens en exigeant que la prestation et le financement privés soient étendus à ces services, notamment à l'adduction d'eau en milieu urbain. L'Accord général sur le commerce des services (AGCS) établi par l'OMC encourage lui aussi une privatisation partielle. Dans le cadre de ces accords, les pays doivent s'engager à «élever progressivement le niveau de libéralisation » et ils sont encouragés avec de plus en plus d'insistance à libéraliser de nouveaux aspects de la fourniture des services.

Il est important de rappeler que la multiplication des partenariats public-privé n'est en rien le résultat logique et inévitable du retour à la rationalité économique des forces de marché. Ils ont été, ces dernières années, institutionnalisés, protégés et guidés par les acteurs dominants dans le cadre de la nouvelle architecture globale en charge du bon fonctionnement de l'économie mondialisée. En 2002, la Conférence des Nations unies sur le financement du développement de Monterrey et le Sommet mondial des Nations unies sur le développement durable de Johannesburg ont placé ces nouveaux partenariats au cœur des transformations de la coopération au développement, en en faisant un des 
nouveaux débouchés les plus prometteurs en termes d'impact sur les conditions de vie des populations.

La Banque mondiale, en association avec d'autres organismes internationaux, a mis en place des dispositifs novateurs afin d'assurer un rôle croissant du secteur privé dans la gestion des services essentiels. Les garanties de la Banque mondiale, la Société financière internationale et l'Agence multilatérale de garantie des investissements aident ainsi les gouvernements à attirer les investisseurs privés dans le secteur de l'eau et l'assainissement grâce à l'atténuation des risques liés à la performance des gouvernements. La Banque mondiale fournit aussi des prêts visant à financer des projets qui respectent les conditions de privatisation et de recouvrement des coûts. C'est le cas, par exemple, du projet de fourniture d'eau à Luanda, en Angola, dont les objectifs sont notamment de recruter des entreprises privées pour gérer le système de distribution d'eau dans les principaux centres urbains et d'adopter un système tarifaire permettant le recouvrement des coûts par la société de distribution de l'eau.

Cette nouvelle vision dominante de la gestion des services essentiels marque aussi le Nouveau Partenariat pour le développement de l'Afrique (NEPAD), lancé en 2001, qui encourage les partenariats entre le secteur public et le secteur privé pour attirer de nouveaux investissements dans différents secteurs essentiels, dont l'eau. Cette position marque un changement profond chez les élites dominantes africaines, qui jouent le jeu de la transnationalisation en ouvrant leurs portes aux investissements directs étrangers des firmes multinationales et en leur proposant des opportunités de valorisation du capital dans de nouveaux secteurs d'activités (transport, énergie, eau ou encore assainissement).

On compte aujourd'hui plus de 2350 partenariats public-privé dans les secteurs de l'eau et de l'assainissement alors qu'ils étaient quasiment inexistants au début des années 1990 (UNDP 2003:116). La promotion des partenariats et la redéfinition des champs de responsabilité public-privé ne sont toutefois pas sans poser de problèmes car sous le couvert d'améliorer la qualité et l'accès aux services essentiels, ces politiques se sont traduites par des résultats parfois controversés lorsqu'ils ont été associés à la privatisation de ces services. Le problème se pose à chaque fois que les sociétés privées ne souhaitent pas assurer l'approvisionnement en eau des campagnes ou des quartiers populaires dans les pays à faible revenu, considérant cette activité comme non rentable compte tenu de l'absence de marchés solvables dans ces zones (SAPRIN 2004). Par exemple, à Cartagena, en Colombie, un grand bidonville n'a pas été raccordé au réseau parce que le prestataire a estimé qu'il ne faisait pas partie de l'agglomération.

Ces partenariats affichent donc des résultats mitigés car, contrairement à ce qui était prévu, la privatisation n'a pas permis de lever les nouveaux capitaux escomptés et les nouveaux partenariats public-privé n'ont pu mobiliser ces ressources supplémentaires pour améliorer la qualité et l'accès à ces services essentiels. Par ailleurs, les plus démunis font les frais de la réticence des opérateurs privés à financer des investissements peu rentables dans les zones populaires et doivent faire face au principe du paiement des services par le client, principe sur lequel reposent les partenariats public-privé. En 2000, dans la ville bolivienne de Cochabamba, par exemple, le prix de l'eau est devenu entre trois et quatre fois plus élevé quelques semaines seulement après qu'une entreprise privée londonienne eut obtenu le marché de l'alimentation en eau de la ville (UNDP 2003 : 117). 
Ces problèmes mettent en lumière les limites des partenariats public-privé si l'on veut maintenir l'idée d'accès universel à ces services essentiels. La prise de conscience des risques d'exclusion des couches populaires de l'accès à ces services essentiels est à la base de la réflexion sur les biens publics globaux, qui ne sont pas des biens comme les autres et doivent à ce titre être fournis par des mécanismes échappant à la logique du marché ou alors par des mécanismes modifiés de marché assurant que nul n'en soit exclu (Kaul, Grunberg et Stern 1999). L'apparition de la notion de responsabilité sociale de l'entreprise a fait suite à la montée des inquiétudes au sujet de ce vaste processus de privatisation de services essentiels. Elle signifie que l'on inclut un nouveau type de responsabilité, qui serait une responsabilité sociale, et que l'on considère l'entreprise non plus comme une entité économique poursuivant le seul objectif de maximisation du profit mais aussi comme une partie prenante parmi d'autres dans son environnement, qui se doit de collaborer à l'amélioration des conditions de vie et de travail des populations tout en préservant sa profitabilité (Hopkins 2004).

Toutefois, on ne peut qu'être prudent par rapport à cette vision a-conflictuelle et a-historique des acquis sociaux, qui met l'accent sur le caractère purement volontaire et non contraignant de ces initiatives de responsabilisation sociale des entreprises alors que l'histoire montre que les acquis sociaux ont toujours été le fruit de luttes intenses. La responsabilité sociale des entreprises ne peut se substituer aux réglementations et législations nationales et internationales régissant les droits sociaux et environnementaux au risque d'une insécurisation des conditions de vie et de travail des populations. Une forte volonté publique est donc nécessaire pour que la privatisation ne se fasse pas au détriment des plus pauvres. En Bolivie, l'eau et l'assainissement à La Paz et El Alto ont été, par exemple, attribués au soumissionnaire qui a promis le plus grand nombre de raccordements dans les quartiers pauvres.

\section{Vers une redéfinition du rôle des populations et de leurs}

\section{organisations représentatives dans la fourniture des services essentiels}

La fourniture de services collectifs par des ONG est aussi considérée comme une voie intermédiaire entre le marché et l'Etat qui a fait ses preuves pour combler les lacunes du système public et pour permettre l'accès des pauvres aux services essentiels. C'est pourquoi on assiste à la multiplication des partenariats entre pouvoirs publics, entreprises et organisations de la société civile. La participation des ONG permet de mieux appréhender les besoins des communautés pauvres. Elles peuvent aussi faire pression pour que ces besoins soient pris en compte par les entreprises privées à travers des campagnes de sensibilisation et de mobilisation.

Le récent rapport du secrétaire des Nations unies sur la société civile exprime parfaitement cette nouvelle vision du développement basée sur la promotion des partenariats public-privé. L'idée centrale est qu'il faut tirer parti des synergies potentielles ou existantes entre les différents acteurs du développement et travailler à mettre en contact les diverses parties prenantes concernées par un problème (Nations unies 2004). La concertation au moment de l'identification des objectifs et de la définition du projet améliore celui-ci, qui s'en trouve enrichi tout en étant plus ancré dans la réalité et en phase avec les demandes de 
développement des populations. De plus, la redéfinition des rôles et des responsabilités des acteurs publics (nationaux ou locaux) et des acteurs privés (secteur privé ou société civile) doit permettre d'améliorer la gestion de toute une série de services essentiels car «là où des capacités sont mises au service de processus associant de multiples parties prenantes, les retombées bénéfiques sont considérables» (Nations unies 2004:11). Ces partenariats constituent dorénavant un outil très important pour la coopération au développement afin de trouver des réponses novatrices à des questions critiques. Les Objectifs du millénaire pour le développement ont aussi eu un effet catalyseur pour les partenariats sur le terrain entre les autorités publiques, le secteur privé et les ONG car la réalisation de ces objectifs nécessite impérativement une action concertée et plurielle.

C'est dans ce contexte que l'on assiste à la participation croissante des ONG dans les organes intergouvernementaux et les divers programmes et projets de développement. La preuve de la viabilité des multipartenariats est venue de la multiplication des initiatives locales et du succès de nombre d'entre elles qui ont réussi à régler des problèmes complexes et à atteindre des objectifs difficiles en mobilisant un grand nombre d'acteurs à tous les stades de l'initiative. A la suite de la reconnaissance du succès d'une grande part de ces nouveaux partenariats, les acteurs non étatiques n'ont plus été considérés comme de simples agents d'exécution mandatés par une institution intergouvernementale ou gouvernementale mais comme des partenaires à part entière de l'élaboration des politiques et de la prise de décision.

L'élargissement de la coopération au développement à une vaste gamme d'acteurs, notamment des acteurs de la société civile ainsi que des pouvoirs publics locaux et des parlementaires, est vue comme «l'occasion de mobiliser des capacités nouvelles et des expériences diverses pour s'attaquer à certains des problèmes les plus exigeants de l'époque» (Cardoso 2004:8). Les recommandations de la Commission Cardoso sont très claires à ce sujet. L'ONU devrait mettre l'accent sur l'inclusion de toutes les parties prenantes concernées tout en ayant conscience que les acteurs clés ne sont pas les mêmes dans tous les cas. Il est essentiel de ce fait d'encourager des partenariats avec différentes parties prenantes pour trouver des solutions efficaces et permettre à toute une gamme de réseaux mondiaux d'innover et de faire avancer les choses (Cardoso 2004: 19).

Le processus de démocratisation et d'ouverture de la coopération au développement à la société civile a été accompagné d'un vaste processus de décentralisation au Sud visant à renforcer les autorités publiques locales comme acteur du développement. Celles-ci sont dorénavant considérées comme des partenaires clés car ce sont elles qui le plus souvent jouent un rôle fondamental au niveau local dans des domaines essentiels pour les conditions de vie des populations comme dans le cas de l'eau, de l'assainissement ou encore des services de santé et l'éducation. La promotion de nouvelles entités publiques locales est préconisée car celles-ci sont souvent capables d'effectuer les investissements nécessaires à un prix nettement moins élevé que le secteur privé, notamment en faisant leurs achats sur place plutôt qu'en les important.

La nouvelle gouvernance mondiale doit donc se construire à partir de la promotion de la participation et de la responsabilité à tous les niveaux. Mais l'engage- 
ment des parties prenantes et le développement des partenariats nécessitent le renforcement des capacités des organisations de la société civile et du secteur privé, des dispositifs de construction de compromis incluant des mécanismes de prévention et de résolution créatrice des conflits, et la formation des fonctionnaires nationaux et multinationaux pour traiter avec une plus large gamme de parties prenantes. Il est nécessaire pour cela de créer un environnement institutionnel propre à susciter ces nouveaux partenariats et à donner aux différentes parties prenantes les moyens d'agir et de mieux développer leur potentiel.

La multiplicité d'acteurs apparaît désormais comme une condition nécessaire des politiques de développement, mais la Commission Cardoso reconnaît cependant qu'il faut se garder de voir dans les partenariats une panacée à tous les problèmes. La notion de partenariat est floue et ne va pas sans poser de nombreux problèmes car elle suppose des degrés d'égalité entre les parties prenantes que l'on trouve rarement dans la pratique. D'autre part, la question de la représentativité des organisations de la société civile est posée tout comme celle de leur responsabilité. Il leur est demandé de justifier leur nouveau statut central dans la coopération au développement. Les politiciens affirment, notamment, être les seuls habilités à parler au nom des citoyens car ils sont démocratiquement élus. Par ailleurs, les dirigeants du monde des affaires peuvent voir d'un mauvais œil les ingérences dans des questions éthiques qui peuvent entrer en contradiction avec les intérêts de leurs actionnaires ou clients.

\section{Les dispositifs de promotion des nouveaux partenariats}

La coopération décentralisée introduite dès 1989 dans le cadre de la Convention de Lomé IV a constitué une nouvelle approche de la coopération au développement. Elle avait pour objectif d'élargir le nombre généralement restreint des acteurs de la coopération en légitimant le rôle des acteurs décentralisés dans le développement (Lapeyre et Fonteneau 1998). Son originalité provenait du soutien qu'elle offrait aux initiatives qui émanaient directement de la base. La responsabilité de la définition des besoins et de la formulation et de la mise en œuvre du projet était transférée aux acteurs locaux. Ceux-ci n'apparaissaient plus comme de simples bénéficiaires ou exécutants de projets définis au-dessus d'eux mais comme les promoteurs de l'amélioration de leurs conditions de vie et de travail.

Le rôle de la coopération décentralisée en tant que nouvel instrument de la coopération au développement a été réaffirmé dans la Convention de Lomé IV bis, qui stipule dans son article 12 bis: «Reconnaissant que les acteurs de la coopération décentralisée peuvent apporter une contribution positive au développement des Etats ACP, les parties contractantes conviennent d'intensifier leurs efforts visant à encourager la participation des acteurs ACP et de la Communauté aux activités de coopération.»

La coopération décentralisée avait pour principal objectif de mieux répondre aux besoins et aux priorités des populations grâce à l'élargissement de la gamme des acteurs du développement à travers l'établissement de nouveaux types de partenariats entre les pouvoirs publics nationaux et locaux, le secteur privé et les organisations de la société civile. L'idée était de promouvoir une redéfinition du rôle 
et des responsabilités de chacun dans le processus de développement en soutenant un processus de responsabilisation des acteurs décentralisés à travers le transfert de responsabilité et une décentralisation des moyens.

Cette nouvelle approche de la coopération au développement sera poussée encore plus loin par la Commission européenne avec l'Accord de Cotonou signé en juin 2000. L'accord comporte notamment des dispositions originales destinées à promouvoir des approches participatives en vue d'assurer la participation de la société civile et des acteurs économiques et sociaux. Conformément aux conditions fixées dans cet accord, les acteurs non étatiques sont informés et impliqués dans la consultation sur les politiques et stratégies de coopération, et sur les priorités de la coopération, en particulier dans les domaines qui les concernent ou qui les affectent directement, ainsi que sur le dialogue politique. Ils reçoivent aussi des ressources financières en vue d'appuyer les processus de développement local. Enfin, ils sont impliqués dans la mise en œuvre des projets et programmes de coopération dans les domaines qui les concernent.

Dans ce cadre, les acteurs non étatiques reçoivent un appui pour le renforcement de leurs capacités dans des domaines critiques en vue d'accroître leurs compétences, en particulier en ce qui concerne les questions organisationnelles, la représentation et la mise en place de mécanismes de consultation dans le but de promouvoir des alliances stratégiques. Afin de répondre aux besoins des collectivités locales en matière de développement, et afin d'encourager tous les acteurs de la coopération décentralisée susceptibles d'apporter leur contribution au développement autonome des Etats ACP, la coopération au développement soutient, d'une part, le financement de microréalisations au niveau local qui ont un impact économique et social sur la vie des populations et, d'autre part, le financement de la coopération décentralisée, en particulier lorsqu'elle associe les efforts et les moyens d'organisation des Etats ACP et de leurs homologues de l'Union européenne.

L'autre grand vecteur de la participation des acteurs non étatiques dans la coopération au développement, ce sont les DSRP (documents de stratégie pour la réduction de la pauvreté), qui ont été introduits fin 1999 à la suite d'une initiative conjointe du FMI et de la Banque mondiale (Banque mondiale 2001; IMF 2003). Ils constituent un nouveau dispositif ayant pour objectif d'aider les pays pauvres et les bailleurs de fonds à renforcer l'impact de leurs efforts communs en matière de lutte contre la pauvreté. Ils sont la matérialisation du nouveau paradigme du développement de l'après-«consensus de Washington ».

L'accès aux ressources des principaux bailleurs de fonds est de plus en plus soumis à la préparation par le pays demandeur d'un tel document spécifiant la situation socio-économique du pays, les objectifs relatifs à la baisse de la pauvreté sur une perspective à long terme et les moyens prévus afin d'atteindre ces objectifs. L'originalité de ce document est qu'il doit être le fruit d'un processus consultatif faisant appel une participation très large des différents acteurs et, en particulier, des acteurs non étatiques. Depuis 1999, le dispositif des DSRP s'est largement propagé dans les pays pauvres. Fin septembre 2003, 14 pays avaient achevé leur DSRP final et 32 pays avaient commencé le leur (IMF 2003). La mise en place des DSRP a constitué une étape importante dans l'évolution de la coopération au développement car ces documents visent à accroître la coordination de l'aide et 
l'harmonisation des procédures des bailleurs de fonds. Ils déterminent les objectifs, priorités et séquences dans la lutte contre la pauvreté à partir desquels l'aide bilatérale et multilatérale va s'organiser.

Les objectifs des DSRP sont multiples mais parmi ceux-ci figurent en bonne place la promotion de la participation des acteurs non étatique à travers la mise en place d'une batterie de techniques participatives et la création d'espaces de dialogue et de concertation. On peut aussi noter la formation de partenariats internes et externes sur des bases nouvelles résultant d'une redistribution plus ou moins importante des rôles et responsabilités entre les différents acteurs du développement: d'une part, entre le gouvernement, les pouvoirs publics locaux, le secteur privé et les acteurs de la société civile; et, d'autre part, entre le gouvernement et les bailleurs de fonds internationaux.

Cependant, ces processus d'élaboration des DSRP apparaissent problématiques, comme le montre un nombre toujours plus grand d'études de cas (Booth 2003; Molenaers et Renard 2002; Piron et Booth 2004). Parmi les problèmes rencontrés, il faut noter le caractère paradoxal du processus d'appropriation du fait que dans tous les cas la mise en place du processus DSRP résulte d'une exigence provenant des organisations internationales. On demande ainsi aux pays pauvres de s'approprier une démarche soigneusement définie à Washington, où la Banque mondiale et le FMI ont fixé les règles à travers un ensemble de notes d'orientation et de documents techniques. C'est donc un processus fortement balisé qui constitue bien une nouvelle forme de conditionnalité. Le rôle dominant des organisations de Bretton Woods dans la hiérarchisation des objectifs et le choix des politiques à mettre en œuvre reste crucial. Le gouvernement est formellement responsable du DSRP mais ce sont la Banque mondiale et le FMI qui vont le valider ou non à travers l'octroi de crédits ou d'allégement de la dette. Ce pouvoir de veto va déterminer la nature des politiques proposées. De plus, le processus de participation est le plus souvent un processus d'information et de consultation qui n'a que très peu d'influence sur le DSRP final.

La richesse des recommandations faites par les acteurs de la société civile est le plus souvent ignorée. Enfin, ce n'est pas l'ensemble de la stratégie de lutte contre la pauvreté qui est ouvert à la participation des acteurs de la société civile car l'objectif prioritaire d'adaptation aux normes de l'économie mondialisée n'est en aucun cas discutable. C'est pourquoi on peut constater que les DSRP n'ont pas ouvert la voie à des stratégies alternatives de développement et demeurent, au contraire, quant au fond très proches des programmes d'ajustement structurel antérieurs même si la forme a changé et inclut des procédures participatives (Lapeyre et Yépez 2005). Dans ce contexte, les grands gagnants des DSRP, ce sont les acteurs du secteur privé qui sont légitimés comme acteurs du développement en participant activement aux dispositifs participatifs qui entourent le processus d'élaboration des DSRP. Compte tenu que les DSRP sont construits autour de l'idée de la centralité de la croissance et des forces de marché pour assurer celle-ci, les acteurs du secteur privé voient leur rôle accru avec la promotion de politiques favorisant les forces de marché, l'accumulation et les partenariats public-privé. 
Dans les DSRP, la participation des acteurs s'inscrit dans les objectifs de croissance et de libéralisation des conditions de l'accumulation tels que fixés par les élites porteuses du projet d'intégration dans l'économie mondiale (Peemans 2002:311). Il s'agit là d'une donnée intangible, et la participation ne peut dès lors que signifier une meilleure information pour susciter l'adhésion des populations à des objectifs prédéterminés par ces élites. Tout le paradoxe de la nouvelle approche des organisations de Bretton Woods tient dans le fait qu'elles prétendent donner la parole aux pauvres alors que, dans le même temps, elles ne permettent pas que leurs revendications et leurs initiatives s'écartent de la voie qu'elles ont soigneusement balisée (Lapeyre 2002).

La nature stratégique des DSRP apparaît clairement si l'on reconnait que la promotion des nouveaux partenariats public-privé - et donc d'un rôle accru des firmes multinationales - vise à promouvoir dans la gestion des services essentiels une logique économique et technique (la priorité donnée aux notions de coût et d'efficacité) au détriment d'autres logiques portées par d'autres acteurs. L'intégration à la marge des organisations populaires dans les dispositifs participatifs fixés par les organisations de Bretton Woods a pour but de les entraîner sur un terrain nouveau, de changer leur logique de fonctionnement et de vaincre leurs résistances. Intégrer le dispositif participatif, c'est d'abord en accepter les règles du jeu et les relations de pouvoir. La relation de pouvoir ne se traduit plus seulement par le fait de donner des ordres. Elle passe désormais par un travail de persuasion, d'information et de formation ayant pour objectif de faire partager à des groupes d'acteurs l'analyse d'une situation et de les orienter vers un nouveau monde d'action et de réaction. Cette régulation de contrôle d'un type nouveau traduit une évolution dans l'exercice du pouvoir des acteurs dominants (Reynaud 1997).

Les DSRP matérialisent un discours d'acteurs dominants qui veulent réaménager les différents niveaux de pouvoir de façon à assurer l'adhésion d'un certain nombre d'acteurs locaux et nationaux aux objectifs présélectionnés dans le cadre du projet d'intégration et à renforcer ainsi la légitimité de ces derniers. Ils sont l'expression de rapports de force qui doivent être rapportés à des logiques d'acteurs bien réels. La promotion des partenariats public-privé doit être vue comme une composante d'une stratégie globale des acteurs dominants visant à favoriser les conditions de l'accumulation dans l'économie mondialisée et à les libérer des contraintes sociales qui pesaient sur elles.

Mais la relation de pouvoir n'est pas figée. L'introduction de dispositifs participatifs, aussi insatisfaisants soient-ils, peut potentiellement favoriser l'émergence de sujets actifs, capables de mettre en doute les initiatives qui leur sont proposées. Les DSRP, en permettant aux organisations de la société civile de disposer de plus d'informations et de moyens de discussion, ne transforment évidemment pas les rapports de force mais ils modifient les ressources disponibles et leur répartition. Ces changements peuvent contribuer à changer les relations de négociation et donner aux acteurs populaires plus de moyens de prendre dans leurs propres mains la sécurisation de leur accès à ces services essentiels. Le non-dit dans l'approche fonctionnelle de la participation par les organisations de Bretton Woods, c'est que la distribution du pouvoir d'initiative n'est jamais 
égale et que l'on ne s'en empare pas librement. C'est pourquoi le réel enjeu en termes de développement, c'est l'appropriation par les organisations populaires du dispositif participatif lui-même afin de changer les règles du jeu définissant qui a le pouvoir de proposition et d'initiative.

\section{Rôle des acteurs populaires}

Mettre l'accent sur les nouveaux partenariats et la participation de la société civile peut permettre de redonner un statut d'acteur du développement à toute une série d'acteurs qui avaient été enfermés dans la catégorie des «pauvres» passifs. C'est finalement ouvrir les yeux sur une autre réalité du développement, dévoilée par un nombre toujours plus grand de travaux qui montrent au quotidien la capacité d'initiative des populations face à la crise de l'économie et de l'Etat (Peemans 2002; Scott 1985; Monnier et Droz 2005). Malgré les nouvelles formes de précarisation et d'exclusion endogènes de la mondialisation de l'économie, il ne faut pas conclure que les populations subissent passivement cet état de chose. C'est donc précisément dans ces espaces sociaux de précarité qui se multiplient que les individus sont susceptibles de redonner du sens à leurs conduites et de réélaborer des liens et des échanges avec les autres.

Ce déplacement radical du regard vers ces «acteurs oubliés » - c'est-à-dire ceux pendant longtemps considérés comme les non-acteurs du développement - doit amener à une tout autre conception des enjeux actuels des politiques de développement et, par conséquent, de la coopération au développement. On ne peut, en effet, que constater le décalage qui existe très souvent entre, d'une part, les demandes de développement des populations telles qu'elles sont révélées à travers la multitude des pratiques populaires et, d'autre part, les priorités de développement définies par les acteurs dominants.

Les quartiers populaires des grandes villes du Sud ne constituent pas, par exemple, un lieu de désorganisation sociale et d'anomie mais bien un espace vivant où la pauvreté et la marginalité n'excluent pas l'action. Les populations en situation précaire sont aussi capables de se mobiliser collectivement pour agir et réinventer en permanence le lien social sur lequel repose la sécurisation de leurs conditions de vie. A travers des modalités variées marquées par la diversité culturelle et sociale, elles expriment de manière plus ou moins inventive leur insertion dans le tissu social et leur participation à la production de leur lieu de vie. Il est donc nécessaire de reconnaître la capacité de ces acteurs de concevoir le monde et leurs projets de développement en fonction de leur identité culturelle, de leur histoire et de leur lieu de vie. Ces pratiques émanant de la base, que l'on pourrait qualifier de «populaires», combinent des stratégies individuelles, des formations de réseaux et des constructions associatives plus ou moins élaborées.

La reconnaissance de la pluralité des acteurs du développement doit nous amener à reconnaître la pluralité des solutions du problème de l'accès aux services essentiels. Ainsi, même si l'on considère la tendance actuelle à la privatisation, celle-ci aura des conséquences très différentes suivant que l'on privatise au profit des firmes multinationales ou au profit des petits entrepreneurs locaux. Le rôle du secteur informel s'est accru considérablement au Sud à partir des années 1980 car c'est à travers lui que les populations ont pu faire face à l'insécurisation de 
leurs conditions de vie et au retrait de l'Etat. Il matérialise la multitude de pratiques économiques populaires qui structurent l'espace de vie des populations et leur assurent tant bien que mal revenu, emploi et accès aux services essentiels. Mais contrairement à la logique de fonctionnement des firmes multinationales, ces petits producteurs locaux sont ancrés dans le «petit marché» local. Celui-ci est un vrai marché mais il est profondément encastré dans les réalités sociétales et il est marqué par la combinaison des logiques de marché, de redistribution et de lien social (Peemans 2002:386). L'accumulation y est encore soumise à des régulations sociales fortes associées à la relation de dépendance-interdépendance qui lie les populations et les petits producteurs locaux. Soutenir ce type d'opérateurs privés, c'est donc favoriser certaines pratiques économiques qui ne sont pas dominées complètement par la logique de l'accumulation et promouvoir un autre modèle de développement que celui porté par les firmes multinationales et les élites transnationales.

Par ailleurs, face à la crise du secteur public au Sud, les populations ne sont pas restées inactives et se sont mobilisées afin de sécuriser leur cadre de vie. On a ainsi assisté à des exemples très intéressants de «privatisation par le bas» (en opposition à la «privatisation par le haut» décidée par les élites nationales et internationales), qui sont de véritables initiatives collectives dans le domaine de l'eau, de l'électricité, du logement ou encore des soins de santé de base. L'exemple du dahoulage ${ }^{l}$ à Bukavu, dans la République démocratique du Congo, est en cela très instructif (Muhinduka 2004). La dégradation du réseau de la Société nationale d'électricité (SNEL) à Bukavu s'est traduite dans les années 1990 par des délestages de plus en plus fréquents et des problèmes d'accès à l'électricité dans les quartiers populaires. C'est dans ce contexte que l'on a assisté au développement de pratiques collectives ayant pour but d'assurer l'accès des acteurs populaires à l'électricité. Le dahoulage constitue une forme de réponse populaire à la question de l'accès à un service essentiel pour les couches sociales les plus défavorisées.

Compte tenu de la faible solvabilité des usagers potentiels des quartiers populaires, de leur incapacité à faire face à des dépenses mensualisées et du coût individuel de raccordement, l'accès de ceux-ci au service de la SNEL est fortement improbable. Dans ce contexte, des communautés locales sur des lieux de vie donnés s'organisent et leurs membres se cotisent pour raccorder un foyer au réseau. A partir de ce foyer, des fils électriques sont tirés pour alimenter les différents membres qui ont cotisé et qui vont payer en fonction de leur consommation. On voit donc émerger des organisations communautaires facilitatrices d'abonnement mais aussi gendarmes de l'électricité - les conflits sont nombreux - et facilitatrices du dahoulage en marchandant et négociant avec les agents de la SNEL pour le détournement de l'électricité. Mais lorsque la chaîne du dahoulage s'allonge, l'intensité de l'électricité tend à baisser et les transformateurs à surchauffer. On voit alors les communautés locales prendre en mains la gestion de ce service essentiel en se cotisant pour acheter un transformateur plus puissant, puis en traitant avec la SNEL pour un protocole d'accord leur permettant d'assurer leur accès à l'électricité. Ce sont donc bien d'autres formes de

Le dahoulage est un néologisme couramment utilisé dans la ville de Bukavu. Dans la culture locale, il signifie approvisionnement libre et obligatoire en feu chez le voisin pour le ménage qui en manquerait. 
partenariat qui se dessinent à la base. En réinventant du lien social, ceux-ci s’inscrivent dans de véritables dynamiques de développement.

\section{En guise de conclusion}

L'idée des partenariats public-privé et de la participation des acteurs non étatiques dans la gestion des services essentiels s'est imposée progressivement dans la coopération au développement dans les années 1990 mais, comme nous l'avons vu, ces partenariats peuvent se construire sur des logiques très différentes: logique de l'accumulation lorsque l'on privilégie les opérateurs du «grand marché » - les multinationales - ou logique du lien social lorsque l'on privilégie les organisations populaires, mais aussi logique hybride dans le cas des opérateurs locaux du petit marché dont les pratiques économiques sont encastrées dans un ensemble de structures sociales en équilibre précaire et en perpétuelle redéfinition.

La reconnaissance récente de l'importance de la participation traduit la reconnaissance de la pluralité des acteurs du développement et de leurs stratégies. Un des plus grands dangers pour toutes les initiatives de changement, ce n'est pas seulement la révolte ou la résistance active, c'est aussi l'indifférence, la possibilité que les populations cibles se tiennent sur leur quant-à-soi et disent aux initiateurs «Cause toujours». Si l'on veut mobiliser les populations dans de réels partenariats visant à améliorer leurs conditions de vie et de travail, l'objectif est de mettre en place un environnement institutionnel offrant un cadre approprié pour la négociation et la construction d'un compromis autour d'un projet global coopératif de développement; ainsi pourra s'ébaucher une capacité d'action collective génératrice d'apprentissage collectif et de lien social. Une telle approche forte de la participation doit s'inscrire dans un projet plus global de démocratie substantive garantissant les droits à l'autoexpression et à l'autodétermination de la collectivité quant à son projet de développement sur son lieu de vie.

Loin de la vision aconflictuelle en termes de parties prenantes (stakeholders) qui marque le discours dominant sur les nouveaux partenariats, ce papier a cherché à montrer la nécessité de reconnaître le caractère central du conflit, de la négociation et du compromis. La notion de conflit permet de découvrir l'acteur collectif qui explicite son projet et confronte ses points de vue, s'affronte à d'autres acteurs, travaille à construire de l'accord et du compromis, à préserver son autonomie et sa marge de manœuvre. Le conflit est un mode normal de fonctionnement; il oblige à rechercher et à formuler une définition commune et mutuellement acceptable de son enjeu à travers la négociation. C'est la condition indispensable à la mise en place de véritables partenariats.

Si les institutions sociales sont obligées d'évoluer et de s'adapter aux nouvelles pressions de l'économie mondialisée, l'Etat reste un acteur central dans le jeu de médiations permettant la préservation du lien social. Les intérêts privés, lorsqu'ils échappent aux formes institutionnelles de contrôle social, sont destructeurs de la cohésion sociale. C'est cette tendance qui avait poussé l'Etat, dans le système capitaliste contemporain, à intervenir activement dans le domaine social. La recherche d'une «troisième voie», sous la forme d'une des 
nombreuses variantes de société démocratique avec une économie mixte, passe par une redéfinition du rôle de l'Etat, qui nécessite que l'on se pose la question de quel Etat et pour quoi faire. La question de l'articulation entre le marché, l'Etat et la société civile est au cœur de la réflexion sur des projets de développement alternatifs viables. La préservation du lien social constitue dans ce cadre une dimension fondamentale du développement.

Dans cette optique, des innovations institutionnelles sont essentielles pour soutenir la multitude de pratiques populaires qui un peu partout au Sud tendent à sécuriser les conditions de vie des laissés-pour-compte des politiques d'intégration et d'ajustement structurel. Il faut toutefois dépasser une approche idyllique des initiatives locales pour essayer de comprendre quelles sont, parmi ces multiples pratiques de résistance, de survie, de solidarité et de sécurisation des conditions de vie, celles qui peuvent être constitutives d'un autre modèle «être et faire ensemble». L'existence bien réelle de ces pratiques ne signifie pas qu'elles opèrent un retournement des processus de précarisation et de marginalisation dont les populations de ces quartiers continuent à subir les contraintes. Il faut insister sur le fait que la subordination ou le poids de la contrainte extérieure n'épuisent pas leur capacité d'initiative. Ces acteurs revendiquent de pouvoir développer des initiatives locales, d'affirmer leur capacité à produire leurs propres règles d'action ou leur volonté de cogérer leur lieu de vie. Ils tentent d'exercer leur autonomie en grignotant des marges de manœuvre ou en négociant, et le contexte actuel leur offre de nouvelles possibilités de mener à bien leurs projets.

\section{Bibliographie}

BANQUE MONDiALE, 1981, Le développement accéléré en Afrique au sud du Sahara, Washington, D.C., Banque mondiale.

_, 2001, Rapport sur le développement dans le monde 2000/2001. Combattre la pauvreté, Paris, ESKA.

Booth, D., 2003, «Introduction and Overview», Development Policy Review, vol. 21, $\mathrm{n}^{\circ} 2$.

CAmdessus, M., 2003, Financer l'eau pour tous, rapport du Panel mondial sur le financement des infrastructures de l'eau, Kyoto, Conseil mondial de l'eau.

CARdoso, F., 2004, Nous, peuples: société civile, Organisations des Nations unies et gouvernance mondiale, rapport du Groupe de personnalités éminentes sur les relations entre l'Organisation des Nations unies et la société civile, New York, Nations unies.

Colclough, C., 1992, «Who Should Learn to Pay?», in Colclough, C., Manor, J. (eds.), States or Markets, London, Clarendon Press.

Coleman, J., 1990, Foundations of Social Theory, Harvard, Harvard University Press.

COMMISSION EUROPÉENNE, 2000, Accord de partenariat entre les membres du groupe des Etats ACP, d'une part, et la Communauté européenne et ses Etats membres, d'autre part, Bruxelles, Commission européenne.

Cornia, G., Jolly, R., Stewart, F. (dir.), 1987, L'ajustement à visage humain. Protéger les groupes vulnérables et favoriser la croissance, trad. de l'anglais par P. Jacquot et P. Tranier, Paris, Economica.

Evans, P., 1995, Embedded Autonomy, Princeton, N.J., Princeton University Press.

—, 1996, «Government Action, Social Capital and Development: Reviewing the Evidence on Synergy », World Development, vol. 24, nº 6 .

Funuyama, F., 1995, Trust: The Social Virtues and the Creation of Prosperity, London, Hamish Hamilton.

Hopkins, M., 2004, Corporate Social Responsibility: An Issues Paper, Working Paper, n 27, Geneva, ILO (International Labour Organization).

IMF (InTERnAtional Monetary Front), 2003, PRSPs: Detailed Analysis of Progress in Implementation, Washington, D.C., International Monetary Fund. 
Isham, J., Narayan, D., Pritchett, L., 1995, «Does Participation Improve Performance? Establishing Causality with Subjective Data», World Bank Economic Review, vol. 9, nº 2.

Jolly, R., EmmeriJ, L., Ghai, D., LAPEYRe, F., 2004, UN Contributions to Development Thinking and Practice, Bloomington, Indiana University Press.

Kaul, I., Grunberg, I., Stern, M., 1999, Global Public Goods, Oxford, Oxford University Press.

Krueger, A., 1990, «Economists' Changing Perceptions of Government», Weltwirtschaftliches Archiv, Band 126, Heft 3, 1990.

LAPEYRE, F., 2002, «Le rêve d'un développement sans conflit», in Rist, G. (dir.), Les mots du pouvoir. Sens et non-sens de la rhétorique internationale, Nouveaux Cahiers de l'IUED, $\mathrm{n}^{\circ} 13$, Genève, Institut universitaire d'études du développement; Paris, Presses Universitaires de France.

LAPEYRE, F., FonteneAu, G., 1998, Les organisations syndicales et la coopération décentralisée, Bruxelles, Confédération européenne des syndicats.

LAPEYRE, F., YÉPEZ, I., 2005, «Les processus participatifs dans les documents stratégiques de réduction de la pauvreté (DSRP). Quelles perspectives pour les pratiques populaires », in MAINGUY, C., GÉrARdin, H., Brot, J., Froger, G., Quels acteurs pour quel développement, Paris, Karthala.

Molenaers, N., Renard, R., 2002, Strengthening Civil Society from Outside? Donor-driven Consultation and Participation Processes in Poverty Reduction Strategies: The Bolivian Case, Working Paper, $n^{\circ}$ 10, Antwerp, Institute of Development Policy and Management, University of Antwerp.

Monnier, L., Droz, Y. (dir.), 2004, Côté jardin, côté cour. Anthropologie de la maison africaine, Nouveaux Cahiers de l'IUED, $\mathrm{n}^{\circ} 15$, Genève, Institut universitaire d'études du développement; Paris, Presses Universitaires de France.

MuHINDUKA, D., 2004, «Les organisations communautaires et l'entreprise publique d'électricité SNEL: la fourniture de l'électricité à Bukavu de 1990 à 2004 », mémoire de DEA, Louvain, Université catholique de Louvain.

NATIONS UNIES, 2004, Rapport du secrétaire général en réponse au rapport du Groupe de personnalités éminentes sur les relations entre l'Organisation des Nations unies et la société civile, doc. A/59/354.

Peemans, J.-P., 1997, «Les enjeux d'une réflexion sur la décentralisation et le développement local en Afrique au sud du Sahara: quelques remarques introductives», papier présenté au colloque «Les dimensions sociales et économiques du développement local et la décentralisation en Afrique au sud du Sahara», 20-21 novembre, Louvain-la-Neuve.

—, 2002, Le développement des peuples face à la modernisation du monde, Paris, Academia; L'Harmattan.

Piron, L.H., Booth, A., 2004, Politics and the PRSP Approach: The Bolivian Case Study, Working Paper, $n^{\circ}$ 238, London, Overseas Development Institute.

Putnam, R., 1993a, Making Democracy Work: Civic Tradition in Modern Italy, Princeton, Princeton University Press.

—, 1993b, «The Prosperous Community», American Prospect, $\mathrm{n}^{\circ} 13$.

ReYNAUd, J.-D., 1997, Les règles du jeu, Paris, Armand Colin.

SAPRIN (Structural Adjustment Participatory Review International Network), 2004, Structural Adjustment, London, Zed Books.

ScotT, J., 1985, Weapons of the Weak, New Haven, Yale University Press.

Stiglitz, J., 1998, Towards a New Paradigm for Development, 9th Raúl Prebisch Lecture, Geneva, UNCTAD.

Tinbergen, J., 1984, «Development Cooperation as a Learning Process», in MeIER, G., SeErs, D. (eds.), Pioneers in Development, Oxford, Oxford University Press.

Tollison, R., 1982, «Rent Seeking: A Survey», Kylos, vol. 35, nº 4.

UndP (United Nations Development Programme), 1990, Human Development Report, New York, United Nations Development Programme.

—, 2003, Human Development Report 2003 : Millennium Development Goals, New York, United Nations Development Programme.

WolfENSOHN, J.D., 1997, «Allocution à la septième séance de la quatre-vingt-cinquième session de la Conférence internationale du travail, 12 juin», compte rendu provisoire, $\mathrm{n}^{\circ} 11$, Genève, Organisation internationale du travail.

—, 2000, «Pour bâtir un monde équitable», discours prononcé devant le Conseil des gouverneurs à Prague (République tchèque), le 26 septembre 2000.

Woolcock, M., 1998, «Social Capital and Economic Development: Toward a Theoretical Synthesis and Policy Framework», Theory and Society, vol. 27, n⿳2 2, pp. 151-208.

World BAnK, 1990, World Development Report 1990 : Poverty, New York, Oxford University Press.

—, 1998, Assessing Aid: What Works, What Doesn't, and Why, World Bank Policy Research Report, Oxford, Oxford University Press. 